\title{
Analysis of L1-Galerkin FEMs for Time-Fractional Nonlinear Parabolic Problems
}

\author{
Dongfang $\mathrm{Li}^{1,2,3}$, Hong-Lin Liao ${ }^{4}$, Weiwei Sun ${ }^{3}$, Jilu Wang ${ }^{5}$ and \\ Jiwei Zhang ${ }^{6, *}$ \\ ${ }^{1}$ School of Mathematics and Statistics, Huazhong University of Science and \\ Technology, Wuhan, 430074, China. \\ ${ }^{2}$ Hubei Key Laboratory of Engineering Modeling and Scientific Computing, Huazhong \\ University of Science and Technology, Wuhan 430074, China. \\ ${ }^{3}$ Department of Mathematics, City University of Hong Kong, Kowloon, Hong Kong. \\ ${ }^{4}$ Department of Mathematics, Nanjing University of Aeronautics and Astronautics, \\ Nanjing, 211106, China. \\ ${ }^{5}$ Department of Scientific Computing, Florida State University, Tallahassee, FL 32306, \\ USA. \\ ${ }^{6}$ Beijing Computational Science Research Center, Beijing 100094, China.
}

Received 3 April 2017; Accepted (in revised version) 25 July 2017

\begin{abstract}
This paper is concerned with numerical solutions of time-fractional nonlinear parabolic problems by a class of $L 1$-Galerkin finite element methods. The analysis of $L 1$ methods for time-fractional nonlinear problems is limited mainly due to the lack of a fundamental Gronwall type inequality. In this paper, we establish such a fundamental inequality for the $L 1$ approximation to the Caputo fractional derivative. In terms of the Gronwall type inequality, we provide optimal error estimates of several fully discrete linearized Galerkin finite element methods for nonlinear problems. The theoretical results are illustrated by applying our proposed methods to the time fractional nonlinear Huxley equation and time fractional Fisher equation.
\end{abstract}

AMS subject classifications: 65M06, 35B65

Key words: Time-fractional nonlinear parabolic problems, L1-Galerkin FEMs, Error estimates, discrete fractional Gronwall type inequality, Linearized schemes.

\section{Introduction}

In this paper, we study numerical solutions of the time-fractional nonlinear parabolic equation

$$
{ }_{0}^{C} \mathcal{D}_{t}^{\alpha} u-\Delta u=f(u), \quad x \in \Omega \times(0, T]
$$

\footnotetext{
*Corresponding author. Email addresses: dfli@hust.edu.cn (D. Li), liaohl@csrc.ac.cn (H.-L. Liao), maweiw@cityu.edu.hk (W. Sun), jwang13@fsu.edu (J. Wang), jwzhang@csrc.ac.cn (J. Zhang)
} 
with the initial and boundary conditions, given by

$$
\begin{array}{ll}
u(x, 0)=u_{0}(x), & x \in \Omega, \\
u(x, t)=0, & x \in \partial \Omega \times[0, T],
\end{array}
$$

where $\Omega \subset \mathbb{R}^{d}(d=1,2$ or 3$)$ is a bounded and convex polygon/polyhedron. The Caputo fractional derivative ${ }_{0}^{C} \mathcal{D}_{t}^{\alpha}$ is defined as

$$
{ }_{0}^{C} \mathcal{D}_{t}^{\alpha} u(x, t)=\frac{1}{\Gamma(1-\alpha)} \int_{0}^{t} \frac{\partial u(x, s)}{\partial s} \frac{1}{(t-s)^{\alpha}} d s, \quad 0<\alpha<1 .
$$

Here $\Gamma(\cdot)$ denotes the usual gamma function.

The model (1.1) is used to describe plenty of nature phenomena in physics, biology and chemistry $[10,15,24,30]$. In the past decades, developing effective numerical methods and rigorous numerical analysis for the time-fractional PDEs have been a hot research spot $[6,8,11,16,25,28,33,35-38]$. Numerical methods can be roughly divided into two categories: indirect and direct methods. The former is based on the solution of an integrodifferential equation by some proper numerical schemes since time-fractional differential equations can be reformulated into integro-differential equations in general, while the latter is based on a direct (such as piecewise polynomial) approximation to the timefractional derivative $[4,5,17,18]$.

Direct methods are widely used in practical computations due to its ease of implementation. One of the most commonly used direct methods is the so-called L1-scheme, which can be viewed as a piecewise linear approximation to the fractional derivative [29] and which has been widely applied for solving various time-fractional PDEs [9,12]. However, numerical analysis for direct methods is limited, even for a simple linear model (1.1) with

$$
f(u)=L_{0} u, \quad t \in(0, T] .
$$

The analysis of L1-type methods for the linear model was studied by several authors, while the convergence and error estimates were obtained under the assumption that

$$
L_{0} \leq 0
$$

in general, see $[13,14,23,31]$. The proof there cannot be directly extended to the case of $L_{0}>0$. Recently, the condition (1.5) was improved in [34], in which a time-fractional nonlinear predator-prey model was studied by an $L 1$ finite difference scheme and $f(u)$ was assumed to satisfy a global Lipschitz condition. The stability and convergence were proved under the assumption

$$
T^{\alpha}<\frac{1}{L \Gamma(1-\alpha)}
$$


Here $L$ denotes the Lipschitz constant. The restriction condition (1.6) implies that the scheme is convergent and stable only locally in time. Similar assumptions appeared in the analysis of L1 type schemes for time-fractional Burger equation [21] and nonlinear Fisher equation [20], respectively, where $L$ may depend upon an upper bound of numerical solutions. In both [20] and [21], a classical finite difference approximation was used for spatial discretization. Several linearized L1 schemes with other approximations in spatial direction, such as spectral methods [2,3] and meshless methods [27], were also investigated numerically for time-fractional nonlinear differential equations. To the authors' knowledge, the analysis of stability and convergence on the fully discrete numerical scheme for nonlinear time-fractional problem has so far received little attention.

It is well known that the classical Gronwall inequality plays an important role in analysis of parabolic PDEs $(\alpha=1)$ and the analysis of corresponding numerical methods also relies heavily on the discrete counterpart of the inequality. Clearly, the analysis of L1-type numerical methods for time-fractional nonlinear differential equations $(0<\alpha<1)$ has not been well done mainly due to the lack of such a fundamental inequality.

The aim of this paper is to present the numerical analysis for several fully discrete L1-Galerkin FEMs for the general nonlinear equation (1.1) with any given $T>0$. The key to our work is to establish such a discrete fractional Gronwall type inequality, i.e., for a positive sequence satisfying

$$
D_{\tau}^{\alpha} \omega^{n} \leq \lambda_{1} \omega^{n}+\lambda_{2} \omega^{n-1}+g^{n}
$$

where $D_{\tau}^{\alpha}$ denotes an $L 1$ approximation to ${ }_{0}^{C} \mathcal{D}_{t}^{\alpha}, \lambda_{1}$ and $\lambda_{2}$ are both positive constants. In terms of the fundamental inequality, we present optimal error estimates of several fully discrete L1-Galerkin FEMs for Eq. (1.1) with linear or nonlinear source $f(u)$. Moreover, our work can be extended to many other direct numerical methods for time-fractional parabolic equations.

The rest of the paper is organized as follows. We present three linearized fully discrete numerical schemes and the main convergence results in Section 2. These schemes are based on an L1 approximation in temporal direction and Galerkin FEMs in spatial direction. In Section 3, a new Gronwall type inequality is established for the $L 1$ approximation and optimal error estimates of the proposed numerical methods are proved. In Section 4, we present numerical experiments on two different models, nonlinear fractional Huley equation and Fisher equation. Numerical examples are provided to illustrate our theoretical analysis. Finally, conclusions and discussions are summarized in Section 5.

\section{L1-Galerkin FEMs and main results}

We first introduce some notations and present several fully discrete numerical schemes.

For any integer $m \geq 0$ and $1 \leq p \leq \infty$, let $W^{m, p}$ be the usual Sobolev space of functions defined in $\Omega$ equipped with the norm $\|\cdot\|_{W^{m, p}}$. If $p=2$, we denote $W^{m, 2}(\Omega)$ by $H^{m}(\Omega)$. Let $\mathcal{T}_{h}$ be a quasiuniform partition of $\Omega$ into intervals $T_{i}(i=1, \cdots, M)$ in $\mathbb{R}^{1}$, or triangles 
in $\mathbb{R}^{2}$ or tetrahedra in $\mathbb{R}^{3}, h=\max _{1 \leq i \leq M}\left\{\right.$ diam $\left.T_{i}\right\}$ be the mesh size. Let $V_{h}$ be the finitedimensional subspace of $H_{0}^{1}(\Omega)$, which consists of continuous piecewise polynomials of degree $r(r \geq 1)$ on $\mathcal{T}_{h}$. Let $\mathcal{T}_{\tau}=\left\{t_{n} \mid t_{n}=n \tau ; 0 \leq n \leq N\right\}$ be a uniform partition of $[0, T]$ with the time step $\tau=T / N$.

Based on a piecewise linear interpolation, the L1-approximation (scheme) to the Caputo fractional derivative is given by

$$
\begin{aligned}
{ }_{0}^{C} \mathcal{D}_{t_{n}}^{\alpha} u & =\frac{1}{\Gamma(1-\alpha)} \sum_{j=1}^{n} \frac{u\left(x, t_{j}\right)-u\left(x, t_{j-1}\right)}{\tau} \int_{t_{j-1}}^{t_{j}} \frac{1}{\left(t_{n}-s\right)^{\alpha}} d s+Q^{n} \\
& =\frac{\tau^{-\alpha}}{\Gamma(2-\alpha)} \sum_{j=1}^{n} a_{n-j}\left(u\left(x, t_{j}\right)-u\left(x, t_{j-1}\right)\right)+Q^{n},
\end{aligned}
$$

where

$$
a_{i}=(i+1)^{1-\alpha}-i^{1-\alpha}, \quad i \geq 0 .
$$

If $u \in C^{2}\left([0, T] ; L^{2}(\Omega)\right)$, the truncation error $Q^{n}$ satisfies $[23,31]$

$$
\left\|Q^{n}\right\|_{L^{2}}=O\left(\tau^{2-\alpha}\right) .
$$

For a sequence of functions $\left\{\omega^{n}\right\}_{n=0}^{N}$, we define

$$
D_{\tau}^{\alpha} \omega^{n}:=\frac{\tau^{-\alpha}}{\Gamma(2-\alpha)} \sum_{j=1}^{n} a_{n-j} \delta_{t} \omega^{j}=\frac{\tau^{-\alpha}}{\Gamma(2-\alpha)} \sum_{j=0}^{n} b_{n-j} \omega^{j}, \quad n=1, \cdots, N,
$$

where $\delta_{t} \omega^{n}=\omega^{n}-\omega^{n-1}$ and

$$
b_{0}=a_{0}, \quad b_{n}=-a_{n-1}, \quad b_{n-j}=a_{n-j}-a_{n-j-1}, \quad j=1, \cdots, n-1 .
$$

With above notations, a linearized L1-Galerkin FEM is: to find $U_{h}^{n} \in V_{h}$ such that

$$
\left(D_{\tau}^{\alpha} U_{h}^{n}, v_{h}\right)+\left(\nabla U_{h}^{n}, \nabla v_{h}\right)=\left(f\left(U_{h}^{n-1}\right), v_{h}\right), \quad \forall v_{h} \in V_{h}, \quad n=1,2, \cdots, N
$$

with $U_{h}^{0}=\Pi_{h} u_{0}$, where $\Pi_{h}$ represents the interpolation operator.

By noting (2.4), we can rewrite the scheme (2.5) equivalently as

$$
\frac{\tau^{-\alpha}}{\Gamma(2-\alpha)} \sum_{j=0}^{n} b_{n-j}\left(U_{h}^{j}, v_{h}\right)+\left(\nabla U_{h}^{n}, \nabla v_{h}\right)=\left(f\left(U_{h}^{n-1}\right), v_{h}\right), \quad \forall v_{h} \in V_{h} .
$$

In this paper, we assume that the function $f: \mathbb{R} \rightarrow \mathbb{R}$ is Lipschitz continuous, i.e.

$$
\left|f\left(\xi_{1}\right)-f\left(\xi_{2}\right)\right| \leq L\left|\xi_{1}-\xi_{2}\right|, \quad \text { for } \quad \xi_{1}, \xi_{2} \in \mathbb{R},
$$

where $L$ denotes the Lipschitz coefficient. We present optimal error estimates of scheme (2.6) in the following theorem and leave the proof in Section 3.1. 
Theorem 2.1. Suppose that the system (1.1)-(1.2) has a unique solution $u \in C^{2}\left([0, T] ; L^{2}(\Omega)\right) \cap$ $C^{1}\left([0, T] ; H^{r+1}(\Omega)\right)$. Then, there exists a positive constant $\tau_{0}$, such that when $\tau \leq \tau_{0}$, the finite element system (2.6) admits a unique solution $U_{h}^{n}, n=1,2, \cdots, N$, satisfying

$$
\left\|u^{n}-U_{h}^{n}\right\|_{L^{2}} \leq C_{0}\left(\tau+h^{r+1}\right),
$$

where $u^{n}=u\left(x, t_{n}\right)$ and $C_{0}$ is a positive constant independent of $\tau$ and $h$.

Remark 2.1. The proof of Theorem 2.1 is based on a Lipschitz condition. If $f \in C^{1}(\mathbb{R})$, Theorem 2.1 still holds. In fact, by using the mathematical induction and inverse inequality $[19,32]$, we have

$$
\left\|U_{h}^{n-1}\right\|_{L^{\infty}} \leq\left\|R_{h} u^{n-1}\right\|_{L^{\infty}}+\left\|R_{h} u^{n-1}-U_{h}^{n-1}\right\|_{L^{\infty}} \leq\left\|R_{h} u^{n-1}\right\|_{L^{\infty}}+C h^{-\frac{d}{2}}\left(\tau+h^{r+1}\right),
$$

where $R_{h}$ denotes the Ritz projection operator. As we can see from (2.9), the boundedness of $\left\|U_{h}^{n-1}\right\|_{L^{\infty}}$ can be obtained while mesh size being small. Therefore, we have

$$
\left\|f\left(u^{n-1}\right)-f\left(U_{h}^{n-1}\right)\right\|_{L^{2}}=\left\|f^{\prime}(\xi)\left(u^{n-1}-U_{h}^{n-1}\right)\right\|_{L^{2}} \leq C\left\|u^{n-1}-U_{h}^{n-1}\right\|_{L^{2}},
$$

where $\xi$ is between $u^{n-1}$ and $U_{h}^{n-1}$. Hence, the results in Theorem 2.1 can be proved by using similar analysis under the assumption $f \in C^{1}(\mathbb{R})$.

We now present two more high-order fully discrete linearized methods.

With the Newton linearized approximation to the nonlinear term, a linearized L1Galerkin FEM is: to find $U_{h}^{n} \in V_{h}$ such that

$$
\left(D_{\tau}^{\alpha} U_{h}^{n}, v_{h}\right)+\left(\nabla U_{h}^{n}, \nabla v_{h}\right)=\left(f\left(U_{h}^{n-1}\right)+f_{1}\left(U_{h}^{n-1}\right)\left(U_{h}^{n}-U_{h}^{n-1}\right), v_{h}\right), \quad n=1, \cdots, N,
$$

where $f_{1}\left(U_{h}^{n-1}\right)=\left.\frac{\partial f}{\partial u}\right|_{u=U_{h}^{n-1}}$.

Moreover, with an extrapolation to the nonlinear term, a linearized L1-Galerkin FEM is: to find $U_{h}^{n} \in V_{h}$ such that

$$
\left(D_{\tau}^{\alpha} U_{h}^{n}, v_{h}\right)+\left(\nabla U_{h}^{n}, \nabla v_{h}\right)=\left(f\left(\widehat{U}_{h}^{n}\right), v_{h}\right), \quad n=1, \cdots, N,
$$

where $\widehat{U}_{h}^{n}=2 U_{h}^{n-1}-U_{h}^{n-2}$ for $n=2, \cdots, N$ and $\widehat{U}_{h}^{1}$ can be obtained by solving the governing equation

$$
\left(D_{\tau}^{\alpha} \widehat{U}_{h}^{1}, v_{h}\right)+\left(\nabla \widehat{U}_{h}^{1}, \nabla v_{h}\right)=\left(f\left(U_{h}^{0}\right)+f_{1}\left(U_{h}^{0}\right)\left(\widehat{U}_{h}^{1}-U_{h}^{0}\right), v_{h}\right) .
$$

We next present the error estimates of schemes (2.10) and (2.11) in the following theorem. 
Theorem 2.2. Suppose that the system (1.1)-(1.2) has a unique solution $u \in C^{2}\left([0, T] ; L^{2}(\Omega)\right) \cap$ $C^{1}\left([0, T] ; H^{r+1}(\Omega)\right)$. Then, there exists a positive constant $\tau_{0}^{*}$, such that when $\tau \leq \tau_{0}^{*}$, the finite element system (2.10) or (2.11) admits a unique solution $U_{h}^{n}, n=1,2, \cdots, N$, satisfying

$$
\left\|u^{n}-U_{h}^{n}\right\|_{L^{2}} \leq C_{0}^{*}\left(\tau^{2-\alpha}+h^{r+1}\right),
$$

where $C_{0}^{*}$ is a positive constant independent of $\tau$ and $h$.

The representation of this paper focuses on the numerical analysis for the linearized scheme (2.6). The analysis for (2.6) can be easily extended to the linearized schemes (2.10) and (2.11). The main difference is that the schemes (2.10) and (2.11) have the convergent order $2-\alpha$ in the temporal direction, while the scheme (2.6) has the order 1.

In the remainder, we denote by $C$ a generic positive constant, which is independent of $n, h, \tau, \tau_{0}, \tau_{0}^{*}, C_{0}$ and $C_{0}^{*}$, but may depend upon $u$ and $f$.

\section{Error analysis}

In this section, we will prove the optimal error estimate given in Theorem 2.1 for proposed scheme (2.6). As we can see below, the following Gronwall type inequality plays a key role in our work. For brevity, we first present the results of the Gronwall type inequality, and leave the proof in Section 3.2.

Lemma 3.1. Suppose that the nonnegative sequences $\left\{\omega^{n}, g^{n} \mid n=0,1,2, \cdots\right\}$ satisfy

$$
D_{\tau}^{\alpha} \omega^{n} \leq \lambda_{1} \omega^{n}+\lambda_{2} \omega^{n-1}+g^{n}, \quad n \geq 1,
$$

where $\lambda_{1}$ and $\lambda_{2}$ are both positive constants independent of the time step $\tau$. Then, there exists a positive constant $\tau^{*}$ such that, when $\tau \leq \tau^{*}$,

$$
\omega^{n} \leq 2\left(\omega^{0}+\frac{t_{n}^{\alpha}}{\Gamma(1+\alpha)} \max _{0 \leq j \leq n} g^{j}\right) E_{\alpha}\left(2 \lambda t_{n}^{\alpha}\right), \quad 1 \leq n \leq N .
$$

Here, $E_{\alpha}(z)=\sum_{k=0}^{\infty} \frac{z^{k}}{\Gamma(1+k \alpha)}$ is the Mittag-Leffler function and $\lambda=\lambda_{1}+\frac{\lambda_{2}}{\left(2-2^{1-\alpha}\right)}$.

\subsection{Proof of Theorem 2.1}

To prove the main results, we first rewrite the system (2.6) as

$$
\frac{\tau^{-\alpha}}{\Gamma(2-\alpha)} b_{0}\left(U_{h}^{n}, v_{h}\right)+\left(\nabla U_{h}^{n}, \nabla v_{h}\right)=\left(f\left(U_{h}^{n-1}\right), v_{h}\right)-\frac{\tau^{-\alpha}}{\Gamma(2-\alpha)} \sum_{j=0}^{n-1} b_{n-j}\left(U_{h}^{j}, v_{h}\right) .
$$

It is obvious that the coefficient matrix of the linear system (3.2) is symmetric and positive definite. Thus, the existence and uniqueness of the solution of the FEM system (2.6) follow immediately. 
We now let $\Pi_{h}$ denote a Lagrange interpolation operator and $R_{h}: H_{0}^{1}(\Omega) \rightarrow V_{h}$ the Ritz projection operator defined by

$$
\left(\nabla\left(v-R_{h} v\right), \nabla v_{h}\right)=0, \quad \text { for all } v_{h} \in V_{h} .
$$

By classical interpolation theory and finite element theories [32], we have

$$
\begin{aligned}
& \left\|v-\Pi_{h} v\right\|_{L^{2}}+h\left\|\nabla\left(v-\Pi_{h} v\right)\right\|_{L^{2}} \leq C h^{s+1}\|v\|_{H^{s+1}}, \\
& \left\|v-R_{h} v\right\|_{L^{2}}+h\left\|\nabla\left(v-R_{h} v\right)\right\|_{L^{2}} \leq C h^{s+1}\|v\|_{H^{s+1}},
\end{aligned}
$$

for any $v \in H_{0}^{1}(\Omega) \cap H^{s+1}(\Omega)$ and $1 \leq s \leq r$.

From (1.1), we can see that the exact solution $u^{n}$ satisfies the following equation

$$
D_{\tau}^{\alpha} u^{n}-\Delta u^{n}=f\left(u^{n-1}\right)+T^{n}
$$

with the truncation error $T^{n}$ given by

$$
T^{n}=D_{\tau}^{\alpha} u^{n}-{ }_{0}^{C} \mathcal{D}_{t_{n}}^{\alpha} u+f\left(u^{n}\right)-f\left(u^{n-1}\right) .
$$

By (2.2), (2.7) and Taylor expansion, we have

$$
\left\|T^{n}\right\|_{L^{2}} \leq C \tau .
$$

Let

$$
e_{h}^{n}=R_{h} u^{n}-U_{h}^{n}, \quad n=0,1, \cdots, N .
$$

Subtracting (3.6) from the numerical scheme (2.6), it is easy to see that $e_{h}^{n}$ satisfies

$$
\left(D_{\tau}^{\alpha} e_{h}^{n}, v_{h}\right)+\left(\nabla e_{h}^{n}, \nabla v_{h}\right)=\left(D_{\tau}^{\alpha}\left(R_{h} u^{n}-u^{n}\right), v_{h}\right)+\left(f\left(u^{n-1}\right)-f\left(U_{h}^{n-1}\right), v_{h}\right)+\left(T^{n}, v_{h}\right)
$$

for any $v_{h} \in V_{h}$ and $n=1,2, \cdots, N$.

Taking $v_{h}=e_{h}^{n}$ in (3.8), we have

$$
\begin{aligned}
& \left(D_{\tau}^{\alpha} e_{h}^{n}, e_{h}^{n}\right)+\left\|\nabla e_{h}^{n}\right\|_{L^{2}}^{2} \\
\leq & \left(\frac{L}{2}+1\right)\left\|e_{h}^{n}\right\|_{L^{2}}^{2}+\frac{L}{2}\left\|e_{h}^{n-1}\right\|_{L^{2}}^{2}+\frac{1}{2}\left\|D_{\tau}^{\alpha}\left(R_{h} u^{n}-u^{n}\right)\right\|_{L^{2}}^{2}+C h^{2(r+1)}+\frac{1}{2}\left\|T^{n}\right\|_{L^{2}}^{2} \\
\leq & \left(\frac{L}{2}+1\right)\left\|e_{h}^{n}\right\|_{L^{2}}^{2}+\frac{L}{2}\left\|e_{h}^{n-1}\right\|_{L^{2}}^{2}+C\left(\tau+h^{r+1}\right)^{2},
\end{aligned}
$$

where we have used (3.7) and

$$
\left\|D_{\tau}^{\alpha} R_{h} u^{n}-{ }_{0}^{C} \mathcal{D}_{t_{n}}^{\alpha} u\right\|_{L^{2}} \leq\left\|D_{\tau}^{\alpha} R_{h} u^{n}-{ }_{0}^{C} \mathcal{D}_{t_{n}}^{\alpha} R_{h} u\right\|_{L^{2}}+\left\|{ }_{0}^{C} \mathcal{D}_{t_{n}}^{\alpha} R_{h} u-{ }_{0}^{C} \mathcal{D}_{t_{n}}^{\alpha} u\right\|_{L^{2}} \leq C \tau^{2-\alpha}+C h^{r+1} .
$$

On the other hand, noting that the coefficients $a_{j}(j=0, \cdots, N)$ defined in (2.1) satisfy

$$
1=a_{0}>a_{1}>\cdots>a_{N}>0,
$$


we obtain

$$
\begin{aligned}
\left(D_{\tau}^{\alpha} e_{h}^{n}, e_{h}^{n}\right) & =\frac{\tau^{-\alpha}}{\Gamma(2-\alpha)}\left(a_{0} e_{h}^{n}-\sum_{j=1}^{n-1}\left(a_{n-j-1}-a_{n-j}\right) e_{h}^{j}-a_{n-1} e_{h^{\prime}}^{0} e_{h}^{n}\right) \\
& \geq \frac{\tau^{-\alpha}}{\Gamma(2-\alpha)}\left(a_{0}\left\|e_{h}^{n}\right\|_{L^{2}}^{2}-\sum_{j=1}^{n-1}\left(a_{n-j-1}-a_{n-j}\right) \frac{\left\|e_{h}^{j}\right\|_{L^{2}}^{2}+\left\|e_{h}^{n}\right\|_{L^{2}}^{2}}{2}-a_{n-1} \frac{\left\|e_{h}^{0}\right\|_{L^{2}}^{2}+\left\|e_{h}^{n}\right\|_{L^{2}}^{2}}{2}\right) \\
& =\frac{\tau^{-\alpha}}{2 \Gamma(2-\alpha)}\left(a_{0}\left\|e_{h}^{n}\right\|_{L^{2}}^{2}-\sum_{j=1}^{n-1}\left(a_{n-j-1}-a_{n-j}\right)\left\|e_{h}^{j}\right\|_{L^{2}}^{2}-a_{n-1}\left\|e_{h}^{0}\right\|_{L^{2}}^{2}\right) \\
& =\frac{\tau^{-\alpha}}{2 \Gamma(2-\alpha)} \sum_{j=0}^{n} b_{n-j}\left\|e_{h}^{j}\right\|_{L^{2}}^{2} \\
& =\frac{1}{2} D_{\tau}^{\alpha}\left\|e_{h}^{n}\right\|_{L^{2}}^{2} .
\end{aligned}
$$

Combining (3.9) and (3.10), we get

$$
D_{\tau}^{\alpha}\left\|e_{h}^{n}\right\|_{L^{2}}^{2} \leq(L+2)\left\|e_{h}^{n}\right\|_{L^{2}}^{2}+L\left\|e_{h}^{n-1}\right\|_{L^{2}}^{2}+2 C\left(\tau+h^{r+1}\right)^{2} .
$$

By Lemma 3.1, there exists a positive constant $\tau^{*}$ such that, when $\tau \leq \tau^{*}$,

$$
\left\|e_{h}^{n}\right\|_{L^{2}} \leq C\left(\tau+h^{r+1}\right)+C\left\|e_{h}^{0}\right\|_{L^{2}} .
$$

With (3.5), the initial error estimate and (3.4), the above estimate further shows that

$$
\left\|u^{n}-U_{h}^{n}\right\|_{L^{2}} \leq\left\|u^{n}-R_{h} u^{n}\right\|_{L^{2}}+\left\|e_{h}^{n}\right\|_{L^{2}} \leq C\left(\tau+h^{r+1}\right) .
$$

Taking $\tau_{0} \leq \tau^{*}$ and $C_{0} \geq C$, the proof of Theorem 2.1 is complete.

\subsection{The proof of Lemma 3.1}

To prove Lemma 3.1, we first present two useful lemmas.

Lemma 3.2. Let $\left\{p_{n}\right\}$ be a sequence defined by

$$
p_{0}=1, \quad p_{n}=\sum_{j=1}^{n}\left(a_{j-1}-a_{j}\right) p_{n-j}, \quad n \geq 1 .
$$

Then it holds that

$$
\begin{aligned}
& \text { (i) } 0<p_{n}<1, \quad \sum_{j=k}^{n} p_{n-j} a_{j-k}=1, \quad 1 \leq k \leq n, \\
& \text { (ii) } \quad \Gamma(2-\alpha) \sum_{j=1}^{n} p_{n-j} \leq \frac{n^{\alpha}}{\Gamma(1+\alpha)}
\end{aligned}
$$


and for $m=1,2, \cdots$,

$$
\text { (iii) } \frac{\Gamma(2-\alpha)}{\Gamma(1+(m-1) \alpha)} \sum_{j=1}^{n-1} p_{n-j} j^{(m-1) \alpha} \leq \frac{n^{m \alpha}}{\Gamma(1+m \alpha)} .
$$

Proof. (i) Since $1 \geq a_{j-1}>a_{j}>0$ for $j \geq 1$, it is easy to verify inductively from (3.12) that $0<p_{n}<1(n \geq 1)$ by mathematical induction. Moreover, we have

$$
\Phi_{n} \equiv \sum_{j=1}^{n} p_{n-j} a_{j-1}=\sum_{j=0}^{n} p_{n-j} a_{j}=\sum_{j=1}^{n+1} p_{n+1-j} a_{j-1}=\Phi_{n+1}, \quad n \geq 1 .
$$

This implies $\Phi_{n}=\Phi_{1}=a_{0} p_{0}=1$ for $n \geq 1$. Substituting $j=l+k-1$, we further find

$$
\sum_{j=k}^{n} p_{n-j} a_{j-k}=\sum_{l=1}^{n-k+1} p_{n-k+1-l} a_{l-1}=\Phi_{n-k+1}=\Phi_{n}=1, \quad 1 \leq k \leq n .
$$

The equality (3.13) is proved.

(ii) To prove (3.14) and (3.15), we introduce an auxiliary function $q(t)=t^{m \alpha} / \Gamma(1+m \alpha)$ for $m \geq 1$. Then for $j \geq 1$, we have

$$
\int_{0}^{j} \frac{(j-s)^{-\alpha} q^{\prime}(s)}{\Gamma(1-\alpha)} d s=\frac{B(m \alpha, 1-\alpha) j^{(m-1) \alpha}}{\Gamma(1-\alpha) \Gamma(m \alpha)}=\frac{j^{(m-1) \alpha}}{\Gamma(1+(m-1) \alpha)},
$$

where we have used the fact that for $z, w>0$

$$
B(z, w) \equiv \int_{0}^{1} s^{z-1}(1-s)^{w-1} d s=\frac{\Gamma(z) \Gamma(w)}{\Gamma(z+w)} .
$$

Let $Q(t)$ be a piecewise linear interpolating polynomial of $q(t)$ satisfying $Q(k)=q^{k}:=$ $q(k)$. Moreover for $j \geq 1$, we define the approximation error by

$$
\int_{0}^{j} \frac{q^{\prime}(s)-Q^{\prime}(s)}{\Gamma(1-\alpha)(j-s)^{\alpha}} d s=\sum_{k=1}^{j} \int_{k-1}^{k} \frac{q^{\prime}(s)-Q^{\prime}(s)}{\Gamma(1-\alpha)(j-s)^{\alpha}} d s:=\sum_{k=1}^{j} R_{k^{\prime}}^{j}
$$

where

$$
R_{k}^{j}=\int_{k-1}^{k} \frac{d[q(s)-Q(s)]}{\Gamma(1-\alpha)(j-s)^{\alpha}}=-\frac{\alpha}{\Gamma(1-\alpha)} \int_{k-1}^{k} \frac{q(s)-Q(s)}{(j-s)^{\alpha+1}} d s, \quad 1 \leq k \leq j .
$$

Combining (3.16) and (3.17) yields

$$
\begin{aligned}
\frac{j^{(m-1) \alpha}}{\Gamma(1+(m-1) \alpha)} & =\frac{1}{\Gamma(1-\alpha)} \sum_{k=1}^{j} \int_{k-1}^{k} \frac{Q^{\prime}(s)}{(j-s)^{\alpha}} d s+\sum_{k=1}^{j} R_{k}^{j} \\
& =\sum_{k=1}^{j} a_{j-k} \frac{\delta_{t} q^{k}}{\Gamma(2-\alpha)}+\sum_{k=1}^{j} R_{k}^{j} .
\end{aligned}
$$


Noting that $q(t)$ is concave (i.e., $\left.q^{\prime \prime}(t) \leq 0\right)$ for $m=1$, we have $Q(t) \leq q(t), R_{k}^{j} \leq 0$ and

$$
1 \leq \sum_{k=1}^{j} a_{j-k} \frac{\delta_{t} q^{k}}{\Gamma(2-\alpha)}
$$

Multiplying (3.19) by $\Gamma(2-\alpha) p_{n-j}$ and summing it over for $j$ from 1 to $n$, we have

$$
\Gamma(2-\alpha) \sum_{j=1}^{n} p_{n-j} \leq \sum_{j=1}^{n} p_{n-j} \sum_{k=1}^{j} a_{j-k} \delta_{t} q^{k}=\sum_{k=1}^{n} \delta_{t} q^{k} \sum_{j=k}^{n} p_{n-j} a_{j-k}=\sum_{k=1}^{n} \delta_{t} q^{k}=\frac{n^{\alpha}}{\Gamma(1+\alpha)}
$$

where we have used the equality (3.13).

(iii) We multiply (3.18) by $\Gamma(2-\alpha) p_{n-j}$ and sum the resulting equality for $j$ from 1 to $n-1$ to obtain

$$
\begin{aligned}
\frac{\Gamma(2-\alpha)}{\Gamma(1+(m-1) \alpha)} \sum_{j=1}^{n-1} p_{n-j} j^{(m-1) \alpha} & =\sum_{j=1}^{n-1} p_{n-j} \sum_{k=1}^{j} a_{j-k} \delta_{t} q^{k}+\Gamma(2-\alpha) \sum_{j=1}^{n-1} p_{n-j} \sum_{k=1}^{j} R_{k}^{j} \\
& =\sum_{k=1}^{n-1} \delta_{t} q^{k} \sum_{j=k}^{n-1} p_{n-j} a_{j-k}+\Gamma(2-\alpha) \sum_{j=1}^{n-1} p_{n-j} \sum_{k=1}^{j} R_{k}^{j} \\
& \leq \sum_{k=1}^{n-1} \delta_{t} q^{k}+\Gamma(2-\alpha) \sum_{j=1}^{n-1} p_{n-j} \sum_{k=1}^{j} R_{k}^{j} \\
& =\frac{(n-1)^{m \alpha}}{\Gamma(1+m \alpha)}+\Gamma(2-\alpha) \sum_{j=1}^{n-1} p_{n-j} \sum_{k=1}^{j} R_{k}^{j} .
\end{aligned}
$$

If $1 \leq m \leq 1 / \alpha, q(t)$ is still concave (i.e., $q^{\prime \prime}(t) \leq 0$ ). Then $R_{k}^{j} \leq 0$ and (3.15) follows immediately from the above estimate.

If $m>1 / \alpha$, by (3.17), we have

$$
\begin{aligned}
R_{k}^{j} & =\int_{k-1}^{k} \frac{(j-s)^{-\alpha}}{\Gamma(1-\alpha)} \int_{k-1}^{k}\left(q^{\prime}(s)-q^{\prime}(\mu)\right) d \mu d s \\
& =\int_{k-1}^{k} \frac{(j-s)^{-\alpha}}{\Gamma(1-\alpha)} \int_{k-1}^{k} \int_{\mu}^{s} q^{\prime \prime}(\eta) d \eta d \mu d s \\
& \leq \int_{k-1}^{k} \frac{(j-s)^{-\alpha}}{\Gamma(1-\alpha)} \int_{k-1}^{k} \int_{\mu}^{k} \frac{d \eta^{m \alpha-1}}{\Gamma(m \alpha)} d \mu d s \\
& =a_{j-k} \int_{k-1}^{k} \frac{k^{m \alpha-1}-\mu^{m \alpha-1}}{\Gamma(2-\alpha) \Gamma(m \alpha)} d \mu, \quad 1 \leq k \leq j .
\end{aligned}
$$


Therefore, by applying (3.13) for $n \geq 1$, we have

$$
\begin{aligned}
\Gamma(2-\alpha) \sum_{j=1}^{n-1} p_{n-j} \sum_{k=1}^{j} R_{k}^{j} & \leq \sum_{j=1}^{n-1} p_{n-j} \sum_{k=1}^{j} a_{j-k} \int_{k-1}^{k} \frac{k^{m \alpha-1}-\mu^{m \alpha-1}}{\Gamma(m \alpha)} d \mu \\
& =\sum_{k=1}^{n-1} \int_{k-1}^{k} \frac{k^{m \alpha-1}-\mu^{m \alpha-1}}{\Gamma(m \alpha)} d \mu \sum_{j=k}^{n-1} p_{n-j} a_{j-k} \\
& \leq \sum_{k=1}^{n-1} \frac{k^{m \alpha-1}}{\Gamma(m \alpha)}-\frac{(n-1)^{m \alpha}}{\Gamma(1+m \alpha)} \\
& \leq \frac{n^{m \alpha}}{\Gamma(1+m \alpha)}-\frac{(n-1)^{m \alpha}}{\Gamma(1+m \alpha)} .
\end{aligned}
$$

Substituting (3.22) into (3.20), the proof of (3.15) is complete.

Definition 3.1. Let $X=\left[v_{1}, x_{2}, \cdots, x_{n}\right]$ and $Y=\left[y_{1}, y_{2}, \cdots, y_{n}\right]$ be two vectors. If $x_{i} \leq y_{i}$ for $i=1,2, \cdots, n$, it is called that $X$ is less than or equal to $Y$, denoted by $X \leq Y$.

Lemma 3.3. Let $\vec{e}=(1,1, \cdots, 1)^{T} \in R^{n}$ and

$$
J=2 \Gamma(2-\alpha) \lambda \tau^{\alpha}\left[\begin{array}{ccccc}
0 & p_{1} & \cdots & p_{n-2} & p_{n-1} \\
0 & 0 & \cdots & p_{n-3} & p_{n-2} \\
\vdots & \vdots & \ddots & \vdots & \vdots \\
0 & 0 & \cdots & 0 & p_{1} \\
0 & 0 & \cdots & 0 & 0
\end{array}\right]_{n \times n} .
$$

Then, it holds that

(i) $J^{i}=0, i \geq n$;

(ii) $J^{m} \vec{e} \leq \frac{1}{\Gamma(1+m \alpha)}\left(\left(2 \lambda t_{n}^{\alpha}\right)^{m},\left(2 \lambda t_{n-1}^{\alpha}\right)^{m}, \cdots,\left(2 \lambda t_{1}^{\alpha}\right)^{m}\right)^{T}, \quad m=0,1,2, \cdots$;

(iii) $\sum_{j=0}^{i} J^{j} \overrightarrow{e^{\prime}}=\sum_{j=0}^{n-1} J^{j} \vec{e} \leq\left(E_{\alpha}\left(2 \lambda t_{n}^{\alpha}\right), E_{\alpha}\left(2 \lambda t_{n-1}^{\alpha}\right), \cdots, E_{\alpha}\left(2 \lambda t_{1}^{\alpha}\right)\right)^{T}, \quad i \geq n$.

Proof. Noting that $J$ is an upper triangular matrix, it is easy to check that (i) holds.

To prove (ii), we apply the mathematical induction. It is obvious that (ii) holds for $m=0$. We assume that (ii) holds for $m=k$. Since $t_{n}=n \tau$ and (3.23), we have

$$
\begin{aligned}
J^{k+1} \vec{e} & =J J^{k} \vec{e} \leq \frac{1}{\Gamma(1+k \alpha)} J\left(\left(2 \lambda t_{n}^{\alpha}\right)^{k},\left(2 \lambda t_{n-1}^{\alpha}\right)^{k}, \cdots,\left(2 \lambda t_{1}^{\alpha}\right)^{k}\right)^{T} \\
& =\frac{\Gamma(2-\alpha)\left(2 \lambda \tau^{\alpha}\right)^{k+1}}{\Gamma(1+k \alpha)}\left(\sum_{j=1}^{n-1} p_{n-j} j^{k \alpha}, \sum_{j=1}^{n-2} p_{n-1-j}(j-1)^{k \alpha}, \cdots, p_{1} 1^{k \alpha}, 0\right)^{T} .
\end{aligned}
$$


By using (3.15) in Lemma 3.2, we further have

$$
\begin{aligned}
J^{k+1} \vec{e} & \leq \frac{\left(2 \lambda \tau^{\alpha}\right)^{k+1}}{\Gamma(1+(k+1) \alpha)}\left(n^{(k+1) \alpha},(n-1)^{(k+1) \alpha}, \cdots, 2^{(k+1) \alpha}, 1^{(k+1) \alpha}\right)^{T} \\
& =\frac{1}{\Gamma(1+(k+1) \alpha)}\left(\left(2 \lambda t_{n}^{\alpha}\right)^{k+1},\left(2 \lambda t_{n-1}^{\alpha}\right)^{k+1}, \cdots,\left(2 \lambda t_{2}^{\alpha}\right)^{k+1},\left(2 \lambda t_{1}^{\alpha}\right)^{k+1}\right)^{T} .
\end{aligned}
$$

Thus (ii) holds for $m=k+1$.

Since (i) implies that $\sum_{j=0}^{i} J^{j} \vec{e}=\sum_{j=0}^{n-1} J^{j} \vec{e}$ for $i \geq n$, and by (ii), we have

$$
\begin{aligned}
\sum_{j=0}^{n-1} J^{j} \vec{e} & \leq \sum_{j=0}^{n-1} \frac{1}{\Gamma(1+j \alpha)}\left(\left(2 \lambda t_{n}^{\alpha}\right)^{j},\left(2 \lambda t_{n-1}^{\alpha}\right)^{j}, \cdots,\left(2 \lambda t_{1}^{\alpha}\right)^{j}\right)^{T} \\
& \leq\left(E_{\alpha}\left(2 \lambda t_{n}^{\alpha}\right), E_{\alpha}\left(2 \lambda t_{n-1}^{\alpha}\right), \cdots, E_{\alpha}\left(2 \lambda t_{1}^{\alpha}\right)\right)^{T} .
\end{aligned}
$$

The proof of Lemma 3.3 is complete.

We now turn back to the proof of Lemma 3.1.

By the definition of L1-approximation (2.3), we get

$$
\sum_{k=1}^{j} a_{j-k} \delta_{t} \omega^{k} \leq \Gamma(2-\alpha) \tau^{\alpha}\left(\lambda_{1} \omega^{j}+\lambda_{2} \omega^{j-1}\right)+\Gamma(2-\alpha) \tau^{\alpha} g^{j}
$$

Multiplying the inequality (3.27) by $p_{n-j}$ and summing over for $j$ from 1 to $n$, we have

$$
\sum_{j=1}^{n} p_{n-j} \sum_{k=1}^{j} a_{j-k} \delta_{t} \omega^{k} \leq \Gamma(2-\alpha) \tau^{\alpha} \sum_{j=1}^{n} p_{n-j}\left(\lambda_{1} \omega^{j}+\lambda_{2} \omega^{j-1}\right)+\Gamma(2-\alpha) \tau^{\alpha} \sum_{j=1}^{n} p_{n-j} g^{j} .
$$

By using the results (3.13) and (3.14) in Lemma 3.2, we obtain

$$
\sum_{j=1}^{n} p_{n-j} \sum_{k=1}^{j} a_{j-k} \delta_{t} \omega^{k}=\sum_{k=1}^{n} \delta_{t} \omega^{k} \sum_{j=k}^{n} p_{n-j} a_{j-k}=\sum_{k=1}^{n} \delta_{t} \omega^{k}=\omega^{n}-\omega^{0}, \quad n \geq 1,
$$

and

$$
\Gamma(2-\alpha) \tau^{\alpha} \sum_{j=1}^{n} p_{n-j} g^{j} \leq \Gamma(2-\alpha) \tau^{\alpha} \max _{1 \leq j \leq n} g^{j} \sum_{j=1}^{n} p_{n-j} \leq \frac{t_{n}^{\alpha}}{\Gamma(1+\alpha)} \max _{1 \leq j \leq n} g^{j}, \quad n \geq 1 .
$$

It follows that

$$
\omega^{n} \leq \Psi_{n}+\Gamma(2-\alpha) \tau^{\alpha} \sum_{j=1}^{n} p_{n-j}\left(\lambda_{1} \omega^{j}+\lambda_{2} \omega^{j-1}\right), \quad n \geq 1,
$$


where

$$
\Psi_{n}:=\omega^{0}+\frac{t_{n}^{\alpha}}{\Gamma(1+\alpha)} \max _{1 \leq j \leq n} g^{j} .
$$

By noting that $\Psi_{n} \geq \Psi_{k}$ for $n \geq k \geq 1$, we get

$$
\omega^{n} \leq 2 \Psi_{n}+2 \Gamma(2-\alpha)\left(\lambda_{1} \tau^{\alpha} \sum_{j=1}^{n-1} p_{n-j} \omega^{j}+\lambda_{2} \tau^{\alpha} \sum_{j=1}^{n} p_{n-j} \omega^{j-1}\right), \quad n \geq 1,
$$

when $\tau \leq \sqrt[\alpha]{\frac{1}{2 \Gamma(2-\alpha) \lambda_{1}}}$.

Let $V=\left(\omega^{n}, \omega^{n-1}, \cdots, \omega^{1}\right)^{T}$. Thus (3.28) can be written in a vector form by

$$
V \leq\left(\lambda_{1} J_{1}+\lambda_{2} J_{2}\right) V+2 \Psi_{n} \vec{e},
$$

where $J_{1}=\frac{1}{\lambda} J$ and

$$
J_{2}=2 \Gamma(2-\alpha) \tau^{\alpha}\left[\begin{array}{ccccc}
0 & p_{0} & \cdots & p_{n-3} & p_{n-2} \\
0 & 0 & \cdots & p_{n-4} & p_{n-3} \\
\vdots & \vdots & \ddots & \vdots & \vdots \\
0 & 0 & \cdots & 0 & p_{0} \\
0 & 0 & \cdots & 0 & 0
\end{array}\right]_{n \times n}
$$

By (3.12), we have

$$
p_{i} \leq \frac{1}{a_{0}-a_{1}} p_{i+1}=\frac{1}{2-2^{1-\alpha}} p_{i+1}, \quad i \geq 0 .
$$

Therefore,

$$
J_{2} V \leq \frac{1}{2-2^{1-\alpha}} J_{1} V
$$

Substituting (3.30) into (3.29), we get

$$
V \leq J V+2 \Psi_{n} \vec{e}
$$

where $J$ is defined in (3.23) with $\lambda=\lambda_{1}+\frac{\lambda_{2}}{2-2^{1-\alpha}}$.

As a result, we see that

$$
\begin{aligned}
V & \leq J V+2 \Psi_{n} \overrightarrow{e^{2}} \leq J\left(J V+2 \Psi_{n} \overrightarrow{e^{2}}\right)+2 \Psi_{n} \overrightarrow{e^{2}}=J^{2} V+2 \Psi_{n} \sum_{j=0}^{1} J^{j} \overrightarrow{e^{2}} \\
& \leq \cdots \leq J^{n} V+2 \Psi_{n} \sum_{j=0}^{n-1} J^{j} \overrightarrow{e^{2}} .
\end{aligned}
$$

By using (i) and (iii) in Lemma 3.3, we obtain (3.1) and complete the proof of Lemma 3.1. 


\section{Numerical examples}

In this section, we present two numerical examples which illustrate the theoretical results given earlier for schemes (2.6), (2.10) and (2.11). The orders of convergence are examined. All the computations are performed by using the software FreeFEM++.

Example 4.1. We first consider the two-dimensional time-fractional Huxley equation

$$
{ }_{0}^{C} \mathcal{D}_{t}^{\alpha} u=\Delta u-u(1-u)^{2}+g_{1}, \quad x \in[0,1] \times[0,1], \quad 0<t \leq 1 .
$$

Eq. (4.1) can describe many different physical models, such as population genetics in circuit theory and the transmission of nerve impulses [20,26]. To obtain a simple benchmark solution, we take the exact solution in the form of

$$
u=\left(1+t^{3}\right)(1-x) \sin (x)(1-y) \sin (y) .
$$

To this end, it is to obtain the source term $g_{1}={ }_{0}^{C} \mathcal{D}_{t}^{\alpha} u-\Delta u+u(1-u)^{2}$.

We apply the linearized schemes (2.6), (2.10) and (2.11) to solve problem (4.1) with linear and quadratic finite element approximations, respectively. Here and below, a uniform triangular partition with $M+1$ nodes in each spatial direction is used. To investigate the temporal convergence rate, we use a quadratic FEM with a fixed spatial mesh size $h=1 / 100$ and several refined time steps $\tau$. Table 1 shows the discrete $L^{2}$-errors at time $T=1$ and convergence rates in temporal direction with different $\alpha$. From Table 1, one can see that the numerical schemes (2.10) and (2.11) have an accuracy of order $2-\alpha$, while numerical scheme (2.6) has an accuracy of order 1.

Table 1: Discrete $L^{2}$-errors $\left\|u^{N}-U_{h}^{N}\right\|_{L^{2}}$ and convergence rates in temporal direction for Eq. (4.1).

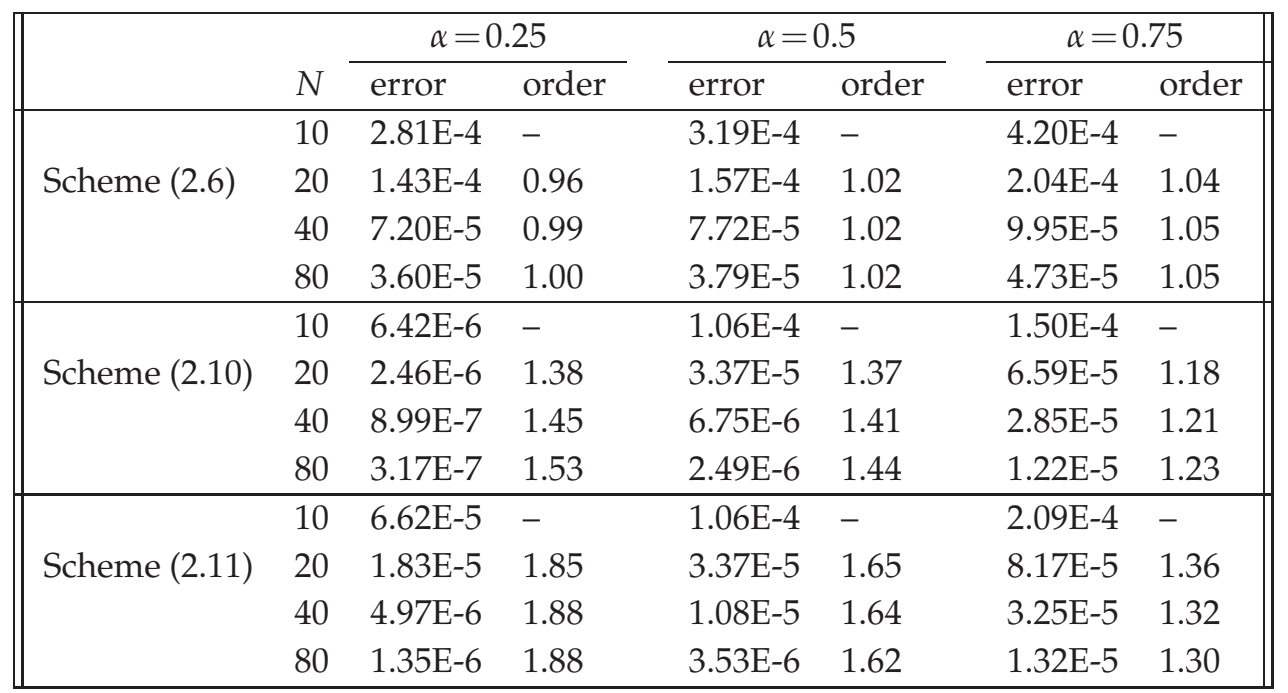


Table 2: Discrete $L^{2}$-errors $\left\|u^{N}-U_{h}^{N}\right\|_{L^{2}}$ and convergence rates in spatial direction for Eq. (4.1).

\begin{tabular}{||llllll||}
\hline \multirow{2}{*}{$M$} & \multicolumn{2}{c}{ L-FEM } & & \multicolumn{2}{c||}{ Q-FEM } \\
\cline { 2 - 3 } \cline { 5 - 6 } & error & order & & error & order \\
\hline 5 & $6.16 \mathrm{E}-3$ & - & & $2.08 \mathrm{E}-4$ & - \\
10 & $1.57 \mathrm{E}-3$ & 1.97 & & $2.61 \mathrm{E}-5$ & 2.99 \\
20 & $3.96 \mathrm{E}-4$ & 1.99 & & $3.26 \mathrm{E}-6$ & 3.01 \\
40 & $9.91 \mathrm{E}-5$ & 2.00 & & $4.08 \mathrm{E}-7$ & 3.00 \\
\hline
\end{tabular}

To investigate the spatial convergence rate, we apply the scheme (2.6) to solve equation (4.1) using both linear and quadratic FEMs with several refined spatial meshes $h$. Table 2 shows the discrete $L^{2}$-errors and convergence rates with $\alpha=0.25$ and $N=M^{3}$. The results in Table 2 indicate that the scheme (2.6) is of optimal convergence order $r+1$ in spatial direction.

Example 4.2. Secondly, we consider the three-dimensional time-fractional Fisher equation

$$
{ }_{0}^{C} \mathcal{D}_{t}^{\alpha} u=\Delta u+u(1-u)+g_{2}, \quad x \in[0,1] \times[0,1] \times[0,1], \quad 0<t \leq 1 .
$$

Eq. (4.2) was originally proposed to describe the spatial and temporal propagation of a virile gene. Later, it is revised by providing some characteristics of memory embedded into the system $[1,20]$. Again, to get a benchmark solution, we calculate the right-hand side $g_{2}$ of (4.2) based on the exact solution

$$
u=t^{2} \sin (\pi x) \sin (\pi y) \sin (\pi z) .
$$

We apply all three proposed schemes with quadratic FEMs to solve Eq. (4.2) by taking $M=60$ and several refined time steps. Table 3 shows the discrete $L^{2}$-errors at time $T=1$ and convergence rates in temporal direction with different $\alpha$. Table 4 shows the discrete $L^{2}$-errors at time $T=1$ and convergence rates in spatial direction for the scheme (2.6) with $\alpha=0.25$ and $N=M^{3}$. Again, the results in Tables 3 and 4 illustrate our theoretical analysis.

\section{Conclusions}

Several linearized L1-Galerkin FEMs have been proposed for solving time-fractional nonlinear parabolic PDEs (1.1) to avoid the iterations at each time step. Error estimates in previous literatures were generally obtained only in a small (local) time interval or in the case that the evolution of the numerical solution decreases in time. In this paper, we established a fundamental Gronwall type inequality for $L 1$ approximation to the Caputo fractional derivative, and provided the theoretical analysis to derive the corresponding optimal error estimates without the restrictions required in previous works. Two numerical examples are given to illustrate our theoretical results. In the future, we will consider 
Table 3: Discrete $L^{2}$-errors $\left\|u^{N}-U_{h}^{N}\right\|_{L^{2}}$ and convergence rates in temporal direction for Eq. (4.2).

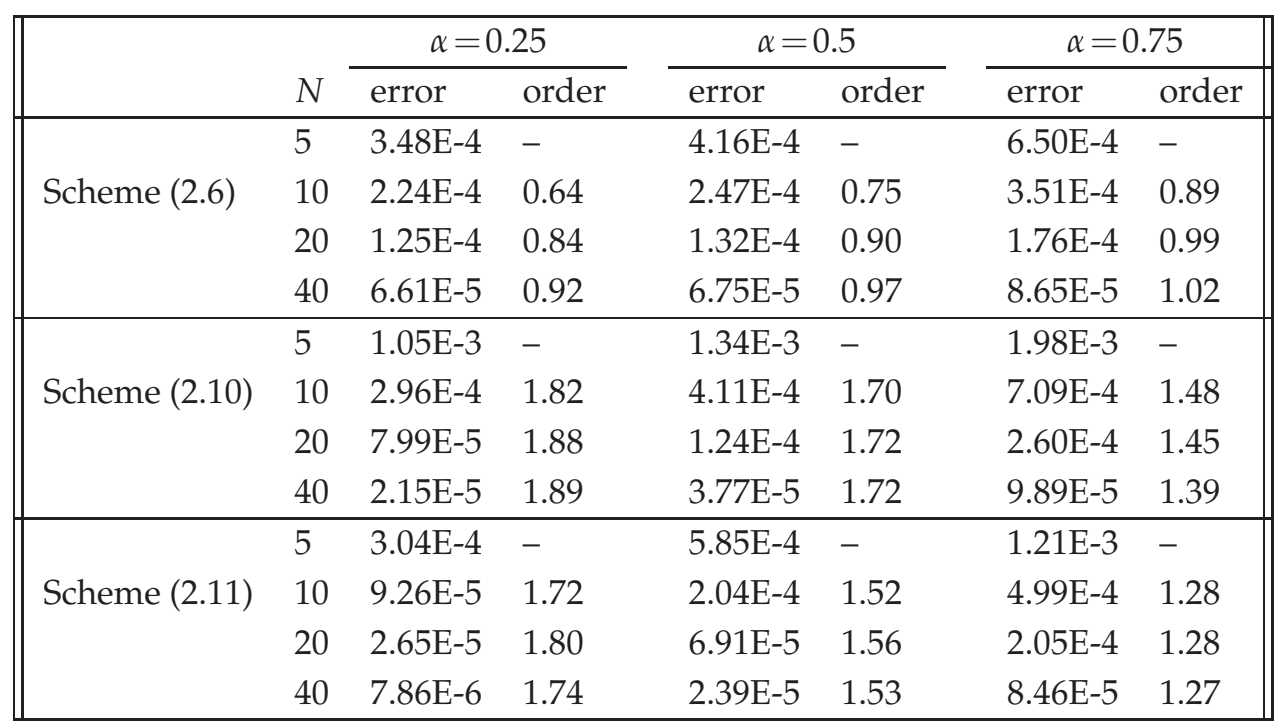

Table 4: Discrete $L^{2}$-errors $\left\|u^{N}-U_{h}^{N}\right\|_{L^{2}}$ and convergence rates in spatial direction for Eq. (4.2).

\begin{tabular}{||llllll||}
\multirow{2}{*}{$M$} & \multicolumn{2}{c}{ L-FEM } & & \multicolumn{2}{c||}{ Q-FEM } \\
\cline { 2 - 3 } \cline { 5 - 6 } & error & order & & error & order \\
\hline 5 & $5.73 \mathrm{E}-2$ & - & & $2.63 \mathrm{E}-3$ & - \\
10 & $1.54 \mathrm{E}-2$ & 1.90 & & $3.27 \mathrm{E}-4$ & 3.01 \\
20 & $3.91 \mathrm{E}-3$ & 1.97 & & $4.09 \mathrm{E}-5$ & 3.00 \\
40 & $9.86 \mathrm{E}-4$ & 1.99 & & $5.11 \mathrm{E}-6$ & 3.00 \\
\hline
\end{tabular}

the sharp error estimate with non-uniform meshes to deal with the singularity of the solution at the initial value for the time fractional reaction-subdiffusion equations [22].

\section{Acknowledgments}

This work is supported by NSFC (Grant Nos. 11771035, 11771162,11571128, 61473126, 91430216, 91530204, 11372354 and U1530401), a grant from the RGC of HK 11300517, China (Project No. CityU 11302915), China Postdoctoral Science Foundation under grant No. 2016M602273, a grant DRA2015518 from 333 High-level Personal Training Project of Jiangsu Province, and the USA National Science Foundation grant DMS-1315259; the USA Air Force Office of Scientific Research grant FA9550-15-1-0001. Jiwei Zhang also thanks the hospitality of Hong Kong City University during the period of his visiting. 


\section{References}

[1] M. Alquran, K. A. Khaled, T. Sardar, J. Chattopadhyay, Revisited Fisher's equation in a new outlook: A fractional derivative approach, Phys. A, 438 (2015), 81-93.

[2] A.H. Bhrawy, E.H. Doha, S.S. Ezz-Eldien, R.A. Van Gorder, A new Jacobi spectral collocation method for solving 1+1 fractional Schrödinger equations and fractional coupled Schrödinger systems, Eur. Phys. J. Plus, 129 (2014), 260.

[3] A.H. Bhrawy, M. A. Abdelkawy, A fully spectral collocation approximation for multidimensional fractional Schrödinger equations, J. Comput. Phys., 294 (2015) 462-483.

[4] J. Cao, C. Xu, A high order schema for the numerical solution of the fractional ordinary differential equations, J. Comput. Phys., 238 (2013), 154-168.

[5] W. Cao, Z. Zhang, G. Karniadakis, Time-splitting schemes for the fractional differential equations I: smooth solutions, SIAM. J. Sci. Comput., 37 (2015), 1752-1776.

[6] C. Chen, F. Liu, V. Anh, I. Turner, Numerical methods for solving a two-dimensional variable-order anomalous subdiffusion equation, Math. Comput., 81 (2012), 345-366.

[7] W. Deng, Numerical algorithm for the time fractional Fokker-Planck equation, J. Comput. Phys., 227 (2007), 1510-1522.

[8] G. Gao and H. Sun, Three-point combined compact alternating direction implicit difference schemes for two-dimensional time-fractional advection-diffusion equations, Commun. Comput. Phys., 17 (2015), 487-509.

[9] G. Gao, Z. Sun, A compact finite difference scheme for the fractional sub-diffusion equations, J. Comput. Phys, 230 (2011), 586-565.

[10] R. Hilfer, Applications of fractional calculus in physics, Word Scientific, Singapore, 2000.

[11] S. Jiang, J. Zhang, Q. Zhang and Z. Zhang, Fast evaluation of the Caputo fractional derivative and its applications to fractional diffusion equations, Commun. Comput. Phys., 21 (2017) 650-678.

[12] Y. Jiang, J. Ma, High-order finite element methods for time-fractional partial differential equations, J. Comp. Appl. Math., 11 (2011), 3285-3290.

[13] B. Jin, R. Lazarov, Z. Zhou, An analysis of the L1 scheme for the subdiffusion equation with nonsmooth data, IMA. J. Numer. Anal., 36 (2016), 197-221.

[14] B. Jin, R. Lazarov, Z. Zhou, Two schemes for fractional diffusion and diffusion-wave equations, SIAM. J. Sci. Comput., 38 (2016), 146-170.

[15] A. A. Kilbas, H. M. Srivastava, J. J. Trujillo, Theory and application of fractional differential equations, Elsevier, Amsterdam, 2006.

[16] T.A.M. Langlands, B.I. Henry, The accuracy and stability of an implicit solution method for the fractional diffusion equation, J. Comput. Phys., 205 (2005), 719-736.

[17] C. Li, C. Tao, On the fractional Adams method, Comp. Math. Appl., 58 (2009), 1573-1588.

[18] C. Li, Q. Yi, A. Chen, Finite difference methods with non-uniform meshes for nonlinear fractional differential equations, J. Comput. Phys., 316 (2016), 614-631.

[19] D. Li, J. Wang, Unconditionally optimal error analysis of Crank-Nicolson Galerkin FEMs for a strongly nonlinear parabolic system, J. Sci. Comput. 72 (2017), 892-915.

[20] D. Li, J. Zhang, Efficient implementation to numerically solve the nonlinear time fractional parabolic problems on unbounded spatial domain, J. Comput. Phys., 322 (2016), 415-428.

[21] D. Li, C. Zhang, M. Ran, A linear finite difference scheme for generalized time fractional Burgers equation, Appl. Math. Model., 40 (2016), 6069-6081.

[22] H. Liao, D. Li, J. Zhang and Y. Zhao, Sharp error estimate of nonuniform L1 formula for time-fractional reaction-subdiffusion equations, Submitted, 2017. 
[23] Y. Lin and C. Xu, Finite difference/spectral approximations for the time-fractional diffusion equation, J. Comput. Phys., 225 (2007), 1533-1552.

[24] R.L. Magin, Fractional calculus in bioengineering, Begell House Publishers, 2006.

[25] W. McLean, K. Mustapha, Convergence analysis of a discontinuous Galerkin method for a sub-diffusion equaiton. Numer. Algor., 52 (2009), 69-88.

[26] M. Merdan, Solutions of time-fractional reaction-diffusion equation with modified Riemann-Liouville derivative, Int. J. Phys. Sci., 7 (2012), 2317-2326.

[27] A. Mohebbi, M. Abbaszadeh, M. Dehghan, The use of a meshless technique based on collocation and radial basis functions for solving the time fractional nonlinear Schrödinger equation arising in quantum mechanics, Eng. Anal. Bound. Elem., 37 (2013), 475-485.

[28] K. Mustapha, H. Mustapha, A second-order accurate numerical method for a semilinear integro-differential equation with a weakly singular kernel, IMA J. Numer. Anal. 30 (2010), 555-578.

[29] K. B. Oldham, J. Spanier, The fractional calculus, Academic Press, New York, 1974.

[30] I. Podlubny, Fractional differential equations, Mathematics in Science and Engineering, Academic Press Inc., San Diego, CA, 1999.

[31] Z.Z. Sun and X. Wu, A fully discrete scheme for a diffusion wave system, Appl. Numer. Math., 56(2) (2006), 193-209.

[32] V. Thomée, Galerkin finite element methods for parabolic problems, Springer-Verlag, 1997.

[33] Y. Yan, Z. Sun and J. Zhang, Fast evaluation of the caputo fractional derivative and its applications to fractional diffusion equations: a second-order scheme. Commun. Comput. Phys. 22 (4), (2017), 1028-1048.

[34] Y. Yu, W. Deng, Y. Wu, Positivity and boundedness preserving schemes for space time fractional predator prey reaction diffusion model, Comput. Math. Appl., 69 (2015), 743-759.

[35] S.B. Yuste, L. Acedo, An explicit finite difference method and a new Neumann-type stability analysis for fractional diffusion equations, SIAM J. Numer. Anal., 42 (2005), 1862-1874.

[36] W. Zhang, C. Li, X. Wu and J. Zhang, High-order local artificial boundary conditions for the fractional diffusion equation on one-dimensional unbounded domain. J. Math. Study. 50 (1) (2017), 28-53.

[37] F. Zeng, C. Li, F. Liu, I. Turner, The use of finite difference/element approaches for solving the time fractional subdiffusion equations, SIAM. J. Sci. Comput., 35 (2013), 2796-3000.

[38] P. Zhuang, F. Liu, V. Anh, I. Turner, Stability and convergence of an implicit numerical method for the nonlinear fractional reaction-subdiffusion process, IMA J. Appl. Math, 74 (2009), 645-667. 